\title{
Cross-tier interference mitigation considering pilot overhead for TDD MIMO heterogeneous networks
}

\author{
Shunsuke Kamiwatari ${ }^{1 a)}$ and Osamu Muta ${ }^{2 b)}$ \\ ${ }^{1}$ Graduate School of Information Science and Electrical Engineering, \\ Kyushu University, 744 Motooka, Nishi-ku, Fukuoka-shi, Fukuoka, Japan \\ ${ }^{2}$ Center for Japan-Egypt Cooperation in Science and Technology, \\ Kyushu University, 744 Motooka, Nishi-ku, Fukuoka-shi, Fukuoka, Japan \\ a)kamiwatari@mobcom.ait.kyushu-u.ac.jp \\ b)muta@ait.kyushu-u.ac.jp
}

\begin{abstract}
In a two-tier heterogeneous network, cross-tier interference mitigation between macro-cell (MC) and small-cell (SC) is an important issue, where MC and SC share the same time/frequency resources. In this article, we propose a method to mitigate downlink cross-tier interference between MC and SC, where macro-cell base-station (MBS) and small-cell base-stations have multiple antenna arrays and their precoding weights are optimized to mitigate mutual cross-tier interference under a given pilot overhead. Simulation results show that the proposed scheme improves the downlink sum-rate compared with conventional scheme that coordinates cross-tier interference from MBS to small-cell users under the same pilot overhead condition.
\end{abstract}

Keywords: two-tier heterogeneous network, massive MIMO, orthogonal pilot, pilot overhead

Classification: Wireless Communication Technologies

\section{References}

[1] F. Zhang, S. Sun, B. Rong, F. R. Yu, and K. Lu, "A novel massive MIMO precoding scheme for next generation heterogeneous networks," IEEE GLOBECOM, San Diego, CA, pp. 1-6, 2015. DOI:10.1109/GLOCOM.2015. 7417346

[2] K. Sundaresan and S. Rangarajan, "Efficient resource management in OFDMA femtocells," Proc. ACM Intl. Symp. Mobile Ad Hoc Netw. Comput., pp. 33-42, May 2009. DOI:10.1145/1530748.1530754

[3] N. Saquib, E. Hossain, L. B. Le, and D. I. Kim, "Interference management in OFDMA femtocell networks: Issues and approaches," IEEE Wireless Commun., vol. 19, no. 3, pp. 86-95, June 2012. DOI:10.1109/MWC.2012.6231163

[4] P. Zhao, Z. Wang, C. Qian, L. Dai, and S. Chen, "Location-aware pilot assignment for massive MIMO systems in heterogeneous networks," IEEE Trans. Veh. Technol., vol. 65, no. 8, pp. 6815-6821, Aug. 2016. DOI:10.1109/ TVT.2015.2480965

[5] W. Hao, O. Muta, H. Gacanin, and H. Furukawa, "Performance analysis on 
uplink pilot allocation in TDD massive MIMO heterogeneous networks," IEICE Trans. Fundamentals, vol. E100-A, no. 11, pp. 2314-2322, Nov. 2017. DOI:10. 1587/transfun.E100.A.2314

[6] T. L. Marzetta, "Noncooperative cellular wireless with unlimited numbers of base station antennas," IEEE Trans. Wireless Commun., vol. 9, no. 11, pp. 3590-3600, Sept. 2010. DOI:10.1109/TWC.2010.092810.091092

\section{Introduction}

Two-tier heterogeneous network (HetNet) is a promising framework for nextgeneration wireless communication systems, where short-range small-cell (SC) base-stations are overlaid on a coverage area of macro-cell (MC) [1]. In particular, in two-tier HetNets, radio spectrum can be utilized more effectively if MC and SC share the same time/frequency resources [2]. However, cross-tier interference coordination between $\mathrm{MC}$ and $\mathrm{SC}$ is necessary to fully harvest potential performance of the HetNets [3, 4, 5].

Adaptive beam-forming using massive multi-input multi-output (mMIMO) technology [6] is an effective approach to mitigate inter-cell interference, e.g., when mMIMO is equipped at MC base station (MBS), the extra spatial degree of freedoms of mMIMO can be utilized to effectively mitigate cross-tier interference from MBS to SC users (SUs) if cross-channel state information (CrCSI) of the path between MC and SC is available. In [4], a location-aware pilot reuse scheme is proposed to coordinate uplink pilot interference. However, downlink cross-tier interference mitigation between MC and SCs is not considered. In [5], a downlink cross-tier interference mitigation scheme was proposed for HetNets with a timedivision-duplex (TDD) mode, where CrCSI is estimated at MBS by overhearing uplink pilots from SUs and designing the precoding weight that cancels downlink cross-tier interference to SUs, where the pilot assignment to SUs is optimized to maximize SUs' sum-rate under a given pilot overhead. Although this scheme is effective in improving SU's rate while minimizing the required pilot overhead, cross-tier interference mitigation from SC base-stations (SBSs) to macro-users (MUs) is not considered.

In this article, based on works in [5], we propose an extended cross-tier interference mitigation scheme that coordinates not only cross-tier interference from MBS to SU but also the interference from SBS to MU in HetNets with TDD where $\mathrm{MMIMO}$ is equipped at MBS.

\section{System model}

We consider a TDD downlink HetNet in Fig. 1, where MBS has massive antenna array with $N_{M}$ elements and serves $M$ single-antenna MUs while each SBS has $N_{S}$ antennas and serves $M_{S}$ SUs. $K$ SBSs are randomly deployed in coverage area of the MC, where $N_{M} \gg N_{S}>M_{S}$ and $N_{M} \gg M$. MBS and SBSs estimate channel state information (CSI) by observing their users' uplink orthogonal pilots and then calculate their downlink precoding weights. 
The received signal at the $m$-th MU is expressed as

$$
\begin{aligned}
y_{m}^{M}= & \underbrace{\sqrt{P_{M} \beta_{0,0, m}^{M, M}} \mathbf{h}_{0,0, m}^{M, M} \mathbf{w}_{m} x_{m}}_{\text {Desired signal }}+\underbrace{\sum_{i=1, i \neq m}^{M} \sqrt{P_{M} \beta_{0,0, m}^{M, M}} \mathbf{h}_{0,0, m}^{M, M} \mathbf{w}_{i} x_{i}}_{\text {Inter-user interference }} \\
& +\underbrace{\sum_{k=1}^{K} \sum_{j=1}^{M_{S}} \sqrt{P_{S} \beta_{k, 0, m}^{S, M}} \mathbf{h}_{k, 0, m}^{S, M} \mathbf{v}_{k, j} s_{k, j}}_{\text {Cross-tier interference }}+\underbrace{n_{m}^{M}}_{\text {Noise }},
\end{aligned}
$$

where $P_{M}$ and $P_{S}$ denote the transit powers of MBS and SBS, respectively. $n_{m}^{M}$ denotes additive white Gaussian noise (AWGN) at $m$-th MU. $\beta_{a, b, c}^{A, B}$ and $\mathbf{h}_{a, b, c}^{A, B}$ $\left(a \in\{0,1,2, \cdots, M\}, b \in\{0,1,2, \cdots, K\}, c \in\left\{0,1,2, \cdots, M_{S}\right\}, A, B \in\{M, S\}\right)$ are pathloss coefficient and fading vector between base stations $A$ and users $B$, respectively. $A=M$ and $A=S$ denote MBS and SBS, respectively. $B=M$ and $B=S$ denote MU and SU, respectively. Here, subscripts $a, b$, and $c$ denote base station index ( $a=0$ : MBS, $a \geq 1$ : SBSs), cell index ( $b=0$ : MC, $b \geq 1$ : SC), and user index per cell. $\mathbf{w}_{i}$ and $x_{i}$ denote $N_{M} \times 1$ precoding vector and transmit data for $i$-th MU, respectively. $\mathbf{v}_{k, j}$ and $s_{k, j}$ are $N_{S} \times 1$ precoding vector and transmit data for $j$-th $\mathrm{SU}$ at the $k$-th $\mathrm{SC}$, respectively.

Similarly, the received signal at $j$-th SU in the $k$-th SC can be written as:

$$
\begin{aligned}
y_{k, j}^{S}= & \underbrace{\sqrt{P_{S} \beta_{k, k, j}^{S, S}} \mathbf{h}_{k, k, j}^{S, S} \mathbf{v}_{k, j} S_{k, j}}_{\text {Desired signal }}+\underbrace{\sum_{i=1, i \neq j}^{M_{S}} \sqrt{P_{S} \beta_{k, k, j}^{S, S}} \mathbf{h}_{k, k, j}^{S, S} \mathbf{v}_{k, i} S_{k, i}}_{\text {Inter-user interference }} \\
& +\underbrace{\sum_{l=1, l \neq k}^{\sum_{n=1}^{M_{S}} \sqrt{P_{S} \beta_{l, k, j}^{S, S}}} \mathbf{h}_{l, k, j}^{S, S} \mathbf{v}_{l, n} S_{l, n}}_{\text {Co-tier interference }}+\underbrace{\sum_{u=1}^{M} \sqrt{P_{M} \beta_{0, k, j}^{M, S}} \mathbf{h}_{0, k, j}^{M, S} \mathbf{w}_{u} x_{u}}_{\text {Cross-tier interference }}+\underbrace{n_{k, j}^{S}}_{\text {Noise }},
\end{aligned}
$$

where $n_{k, j}^{S}$ denotes the AWGN.

Let $\mathbf{H}^{M, M}=\left[\left(\mathbf{h}_{0,0,1}^{M, M}\right)^{T},\left(\mathbf{h}_{0,0,2}^{M, M}\right)^{T}, \cdots,\left(\mathbf{h}_{0,0, M}^{M, M}\right)^{T}\right]^{T} \in \mathbb{C}^{M \times N_{M}}$ denote channel matrix estimated at MBS, where $(\mathbf{a})^{T}$ denotes transpose of a vector a. To mitigate inter-user interference, ZF precoding matrix at MBS, $\mathbf{W} \in \mathbb{C}^{N_{M} \times M}$, is given as

$$
\begin{aligned}
\mathbf{W} & =\left(\mathbf{w}_{1}, \mathbf{w}_{2}, \cdots, \mathbf{w}_{M}\right) \\
& =\frac{1}{\left\|\left(\mathbf{H}^{M, M}\right)^{H}\left\{\mathbf{H}^{M, M}\left(\mathbf{H}^{M, M}\right)^{H}\right\}^{-1}\right\|_{2}^{2}}\left(\mathbf{H}^{M, M}\right)^{H}\left\{\mathbf{H}^{M, M}\left(\mathbf{H}^{M, M}\right)^{H}\right\}^{-1},
\end{aligned}
$$

where $(\cdot)^{H}$ denotes conjugate transpose of a complex matrix. $\|\mathbf{A}\|_{2}$ denotes the Frobenius norm of $\mathbf{A} \in \mathbb{C}$.

Similarly, let $\mathbf{H}_{k}^{S, S}=\left[\left(\mathbf{h}_{k, k, 1}^{S, S}\right)^{T}, \cdots,\left(\mathbf{h}_{k, k, M_{S}}^{S, S}\right)^{T}\right]^{T} \in \mathbb{C}^{M_{S} \times N_{S}}$ denote channel matrix between $k$-th SBS and associated SUs. ZF precoding matrix at $k$-th $\operatorname{SBS} \mathbf{V}_{k} \in$ $\mathbb{C}^{N_{S} \times M_{S}}$ is written as:

$$
\begin{aligned}
\mathbf{V}_{k} & =\left(\mathbf{v}_{k, 1}, \mathbf{v}_{k, 2}, \cdots, \mathbf{v}_{k, M_{S}}\right) \\
& =\frac{1}{\left\|\left(\mathbf{H}_{k}^{S, S}\right)^{H}\left\{\mathbf{H}_{k}^{S, S}\left(\mathbf{H}_{k}^{S, S}\right)^{H}\right\}^{-1}\right\|_{2}^{2}}\left(\mathbf{H}_{k}^{S, S}\right)^{H}\left\{\mathbf{H}_{k}^{S, S}\left(\mathbf{H}_{k}^{S, S}\right)^{H}\right\}^{-1} .
\end{aligned}
$$




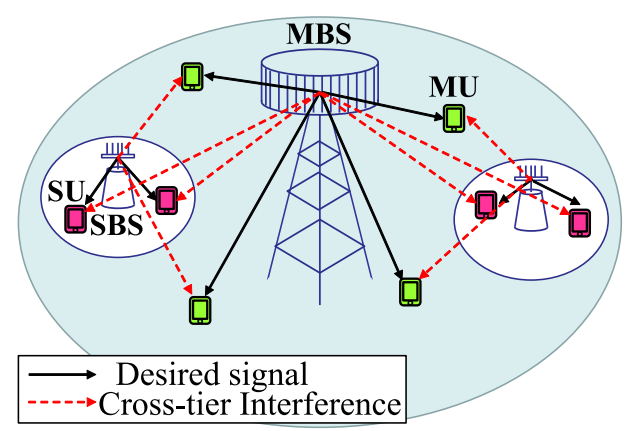

(a) Scenario1

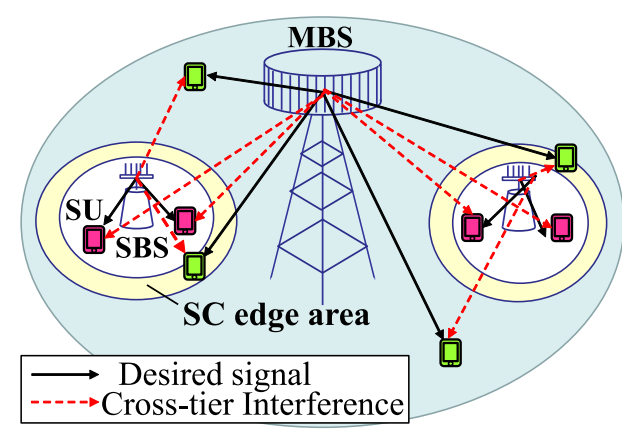

(b) Scenario2

Fig. 1. HetNets system model.

Hence, signal to interference plus noise ratio (SINR) at $m$-th MU and that at $j$-th SU in $k$-th SC, are given below, respectively.

$$
\begin{gathered}
\operatorname{SINR}_{m}^{M}=\frac{P_{M} \beta_{0,0, m}^{M, M}\left|\mathbf{h}_{0,0, m}^{M, M} \mathbf{w}_{m}\right|^{2}}{\sum_{k=1}^{K} \sum_{j=1}^{M_{S}} P_{S} \beta_{k, 0, m}^{S, M}\left|\mathbf{h}_{k, 0, m}^{S, M} \mathbf{v}_{k, j}\right|^{2}+\sigma^{2}}, \\
\operatorname{SINR}_{k, j}^{S}=\frac{P_{S} \beta_{k, k, j}^{S, S}\left|\mathbf{h}_{k, k, j}^{S, S} \mathbf{v}_{k, j}\right|^{2}}{\sum_{l=1, l \neq k}^{K} \sum_{n=1}^{M_{S}} P_{S} \beta_{l, k, j}^{S, S}\left|\mathbf{h}_{l, k, j}^{S, S} \mathbf{v}_{k, j}\right|^{2}+\sum_{u=1}^{M} P_{M} \beta_{0, k, j}^{M, S}\left|\mathbf{h}_{0, k, j}^{M, S} \mathbf{w}_{u}\right|^{2}+\sigma^{2}} .
\end{gathered}
$$

\section{Conventional scheme}

This section explains a pilot-user allocation and cross-tier interference mitigation scheme in [5]. In this scheme, all MUs and $K_{O}$ SUs are allocated orthogonal pilots, where $0 \leq K_{O} \leq K M_{S}$. The other SUs are allocated the same orthogonal pilot, where $K M_{S}$ denotes the total number of SUs. Hence, the number of required orthogonal pilots is $M+K_{O}+M_{S}$. MBS can distinguish not only $M$ MU pilots but also $K_{O}$ SUs' pilot, because they are orthogonal each other. Thus, CSI of the path between MBS and $K_{O}$ SUs can be estimated at MBS by extracting $K_{O}$ SUs' pilots. Hence, cross-tier interference from MBS to $K_{O}$ SUs can be canceled by designing MBS precoding matrix $\mathbf{F} \in \mathbb{C}^{N_{M} \times K_{O}}$ as

$$
\begin{aligned}
\mathbf{F} & =\left(\mathbf{f}_{1}, \mathbf{f}_{2}, \cdots, \mathbf{f}_{K_{O}}\right) \\
& =\frac{1}{\left.\|\left(\mathbf{H}^{M, S}\right)^{H}\left\{\mathbf{H}^{M, S}\left(\mathbf{H}^{M, S}\right)^{H}\right)\right\}^{-1} \|_{2}^{2}}\left(\mathbf{H}^{M, S}\right)^{H}\left\{\mathbf{H}^{M, S}\left(\mathbf{H}^{M, S}\right)^{H}\right\}^{-1},
\end{aligned}
$$

where $\mathbf{H}^{M, S}=\left[\left(\mathbf{h}_{0,0,1}^{M, S}\right)^{T},\left(\mathbf{h}_{0,0,2}^{M, S}\right)^{T}, \cdots,\left(\mathbf{h}_{0,0, K_{O}}^{M, S}\right)^{T}\right]^{T} \in \mathbb{C}^{K_{O} \times N_{M}}$ denotes interference channel matrix, and $\mathbf{h}_{0,0, j}^{M, S}$ denotes CSI of the path between MBS and $j$-th SU. $\mathbf{f}_{j} \in \mathbb{C}^{N_{M} \times 1}$ denotes the precoding vector to cancel cross-tier interference to $j$-th $\mathrm{SU}\left(j \leq K_{O}\right)$. From (3) and (7), the concatenated ZF precoding matrix at MBS, $\mathbf{W}^{M} \in \mathbb{C}^{N_{M} \times\left(K_{O}+M\right)}$, can be written as

$$
\mathbf{W}^{M}=(\mathbf{W}, \mathbf{F})=\left(\mathbf{w}_{1}, \mathbf{w}_{2}, \cdots, \mathbf{w}_{M}, \mathbf{f}_{1}, \mathbf{f}_{2}, \cdots, \mathbf{f}_{K_{O}}\right) .
$$

From (5) and (8), $m$-th MU's rate is lower-bounded as [5] 


$$
R_{\gamma_{m}}^{M}=[1-\underbrace{\frac{K_{O}+M+M_{S}}{L}}_{\text {pilot overhead }}] \log _{2}\left[1+\frac{P_{M} \beta_{0,0, m}^{M, M}\left(N_{M}-M-K_{O}\right)}{\sum_{k=1}^{K} \sum_{j=1}^{M_{S}} P_{S} \beta_{k, 0, m}^{S, M}+\sigma^{2}}\right],
$$

where, $\mathrm{L}$ is the number of symbols per frame. From (6) and (8), $j$-th SU rate that uses the orthogonal pilot is lower-bounded as [5]

$$
R_{\gamma_{k, j}}^{S}=[1-\underbrace{\frac{K_{O}+M+M_{S}}{L}}_{\text {pilot overhead }}] \log _{2}\left[1+\frac{P_{S} \beta_{k, k, j}^{S, S}\left(N_{S}-M_{S}\right)}{\sum_{l=1, l \neq k}^{K} \sum_{n=1}^{M_{S}} P_{S} \beta_{l, k, j}^{S, S}+\sigma^{2}}\right] .
$$

On the other hand, the other SU's rate affected by cross-tier interference from MBS is lower-bounded as [5]:

$$
\begin{aligned}
\hat{R}_{\gamma_{k, j}}^{S}= & {[1-\underbrace{\frac{K_{O}+M+M_{S}}{L}}_{\text {pilot overhead }}] } \\
& \cdot \log _{2}\left[1+\frac{P_{S} \beta_{k, k, j}^{S, S}\left(N_{S}-M_{S}\right)}{\sum_{l=1, l \neq k}^{K} \sum_{n=1}^{M_{S}} P_{S} \beta_{l, k, j}^{S, S}+\sum_{m=1}^{M} P_{M} \beta_{m, k, j}^{M, S}+\sigma^{2}}\right] .
\end{aligned}
$$

It is clear from Eqs (9), (10), and (11) that pilot overhead (i.e., pilot length) increases as $K_{O}$ increases while SINR at each SU is improved by mitigating cross-tier interference. This implies that a trade-off relationship exists between pilot overhead and cross-tier interference mitigation capability.

The downlink sum-rate can be given as

$$
R=\sum_{m=1}^{M} R_{\gamma_{m}}^{M}+\sum_{k, j \in \mathcal{K}_{O}} R_{\gamma_{k, j}}^{S}+\sum_{k, j \notin \mathcal{K}_{O}} \hat{R}_{\gamma_{k, j}}^{S},
$$

where $\mathcal{K}_{O}$ denotes a set of user indices who are allocated orthogonal pilots. The cardinality of $\mathcal{K}_{O}$ is $K_{O}$. The above sum-rate can be maximized by optimizing pilot allocation $\mathcal{K}_{O}[5]^{2}$.

This scheme mitigates cross-tier interference from MBS to SUs while minimizing the required pilot overhead. However, cross-tier interference mitigation from SBS to MUs are not considered.

\section{Proposed scheme}

Based on works in [5], we propose an extended cross-tier interference mitigation scheme that coordinates not only cross-tier interference from MBS to SU but also that from SBS to MUs, where SBS estimates CrCSI of the path between SBS and MUs by overhearing MU's pilots. The proposed scheme can mitigate cross-tier interference from SBS to MUs without changing the pilot allocation (i.e., without increasing the required pilot overhead). 
Similarly to the conventional scheme in [5], we assume that all MUs and $K_{O}$ SUs are allocated the orthogonal pilots and the remaining SUs are allocated the same pilots. Since all MUs' pilots are orthogonal to SUs' ones, each SBS can distinguish MUs' and SUs' pilots. Thus, SBS can estimate cross-channel state information (CrCSI) between SBS and MUs by receiving orthogonal pilots from MUs. More concretely, let $K_{I C}$ denote the number of MUs who are strongly affected by cross-tier interference from SBS. By detecting $K_{I C}$ MUs' pilots, CrCSI between $k$-th SBS and $K_{I C}$ MUs can be estimated as

$$
\mathbf{H}_{k}^{S, M}=\left[\left(\mathbf{h}_{k, 0,1}^{S, M}\right)^{T},\left(\mathbf{h}_{k, 0,2}^{S, M}\right)^{T}, \cdots,\left(\mathbf{h}_{k, 0, K_{I C}}^{S, M}\right)^{T}\right]^{T} \in \mathbb{C}^{K_{I C} \times N_{S}},
$$

where $0 \leq K_{I C} \leq N_{S}-M_{S}$. Hence, the precoding matrix at $k$-th SBS to cancel cross-tier interference to $K_{I C}$ MUs $\mathbf{G}_{k} \in \mathbb{C}^{N_{S} \times K_{I C}}$ can be given as

$$
\begin{aligned}
\mathbf{G}_{k} & =\left(\mathbf{g}_{k, 1}, \mathbf{g}_{k, 2}, \cdots, \mathbf{g}_{k, K_{I C}}\right) \\
& =\frac{1}{\left\|\left(\mathbf{H}_{k}^{S, M}\right)^{H}\left\{\mathbf{H}_{k}^{S, M}\left(\mathbf{H}_{k}^{S, M}\right)^{H}\right\}^{-1}\right\|_{2}^{2}}\left(\mathbf{H}_{k}^{S, M}\right)^{H}\left\{\mathbf{H}_{k}^{S, M}\left(\mathbf{H}_{k}^{S, M}\right)^{H}\right\}^{-1},
\end{aligned}
$$

where $\mathbf{g}_{k, m} \in \mathbb{C}^{N_{S} \times 1}$ denotes the precoding vector of $k$-th SBS for $m$-th MU. From (4) and (14), the concatenated precoding matrix at $k$-th SBS, $\mathbf{W}_{k}^{S} \in \mathbb{C}^{N_{S} \times\left(M_{S}+K_{I C}\right)}$, can be given as

$$
\mathbf{W}_{k}^{S}=\left(\mathbf{V}_{k}, \mathbf{G}_{k}\right)=\left(\mathbf{v}_{k, 1}, \mathbf{v}_{k, 2}, \cdots, \mathbf{v}_{k, M_{S}}, \mathbf{g}_{k, 1}, \mathbf{g}_{k, 2}, \cdots, \mathbf{g}_{k, K_{I C}}\right) .
$$

Assuming $N_{S}>M_{S}$, each SBS can cancel $K_{I C}\left(\leq N_{S}-M_{S}\right)$ cross-tier interference at maximum at expense of decreasing the precoding gain to served SUs ${ }^{3}$. More concretely, MUs' sum-rate can be improved by increasing $K_{I C}$ but SU's rate is decreased due to the decreased precoding gain. Thus, it is necessary to find the optimum value of $K_{I C}$ that maximizes the total sum-rate. In this article, each SBS mitigates cross-tier interference to the nearest MU.

\section{Performance evaluation}

We consider a single MC with a radius of 1000 meters and there is no user within 100 meters of MC center area, while the radius of each SC is 30 meters and there is no user within 5 meters of SC center area [5]. The minimum distance between two SBSs is 120 meters [5]. $P_{M}=46[\mathrm{dBm}]$ and $P_{S}=26[\mathrm{dBm}]$. Noise power is $-104[\mathrm{dBm}]$. The other parameters are $N_{M}=70, N_{S}=10, M=18, M_{S}=2$ and $K=13$. The number of symbols per frame is $L=200$. The received signal is affected by pathloss and independent equal-level two-path Rayleigh fading. Pathloss between MBS and MU/SU is $27.3+39.1 \log 10(\mathrm{~d})$, and pathloss between SBS and MU/SU is $36.8+36.7 \log 10$ (d) [5]. We consider two scenarios in Fig. 1. In scenario 1 (Fig. 1(a)), MUs are uniformly distributed in the MC, while scenario 2 (Fig. 1(b)) assumes that one MU is located near each SC and the rest of MUs are uniformly distributed within the $\mathrm{MC}$, where $R_{\text {edge }}$ is 5 meters.

Fig. 2 shows the downlink sum-rate versus $K_{O}$ for the proposed method (solid line) and the conventional method (dotted line) [5]. Here, the conventional method corresponds to $K_{I C}=0$, while the proposed scheme assumes $K_{I C}=1$. In Fig. 2(a),

${ }^{3}$ This article assumes that each user equips single antenna. If each user has $N_{U}$ antennas, the numbers of antennas at MBS and each SBS must be $N_{M} \geq N_{U}\left(M+K_{O}\right)$ and $N_{S} \geq N_{U}\left(M_{S}+K_{I C}\right)$. 


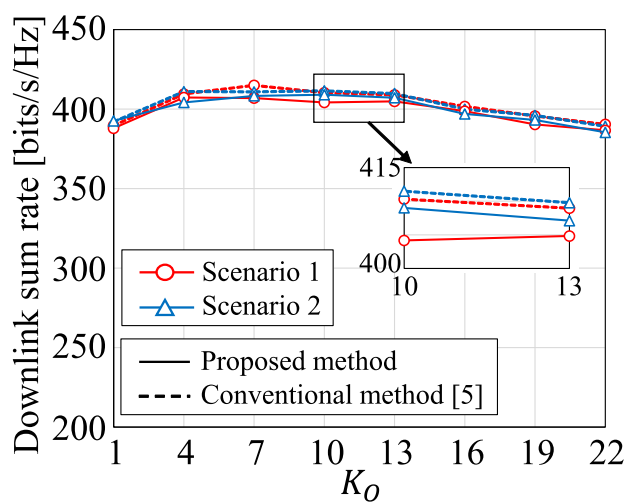

(a) SU's downlink sum rate versus $K_{O}$

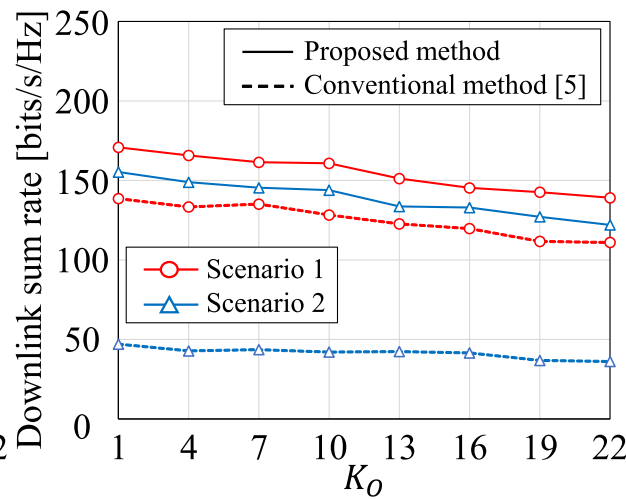

(b) MU's downlink sum rate versus $K_{O}$

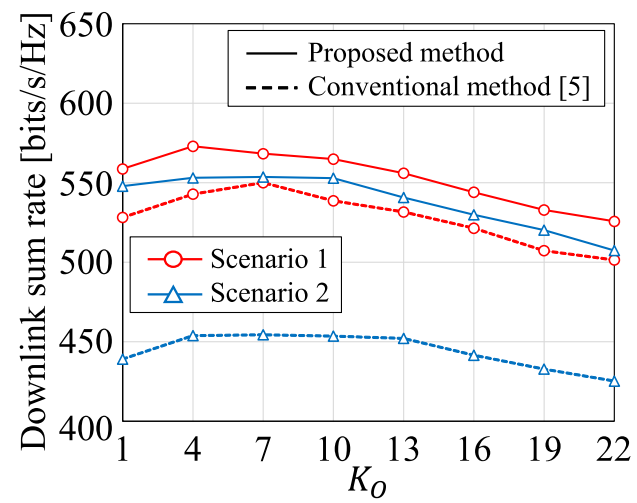

(c) Total downlink sum rate versus $K_{O}$ $((\mathrm{a})+(\mathrm{b}))$

Fig. 2. Downlink sum rate versus $K_{O}$.

there is optimal $K_{O}$ that maximizes SUs' sum-rate. Since SBS uses spatial degree of freedom for mitigating cross-tier interference to MUs, SU's downlink rate slightly decreases in both scenarios. In Fig. 2(b), as $K_{O}$ increases, the downlink rate of MUs decreases monotonously due to the increase of pilot overhead. MU's downlink rate can be improved by applying the proposed scheme especially in scenario 2, because dominant cross-tier interference to MUs is mitigated. In Fig. 2(c), the downlink total sum-rate (i.e., MU rate $+\mathrm{SU}$ rate) can be improved by interference mitigation at SBS. This is because the effect of cross-tier interference mitigation to MUs is higher than the SUs' rate degradation.

Fig. 3 shows downlink sum-rate as a function of $K_{I C}$, where $K_{O}=4$ is chosen as the best value to maximize the achieved sum-rate of the proposed method. In both scenarios, as $K_{I C}$ increases, MU's downlink sum rate is improved monotonously, while SU's sum rate is degraded slightly due to the reduced precoding gain at each SBS. The total sum-rate can be improved by choosing the proper $K_{I C}$ in the propose scheme especially when MU is located near SC. 


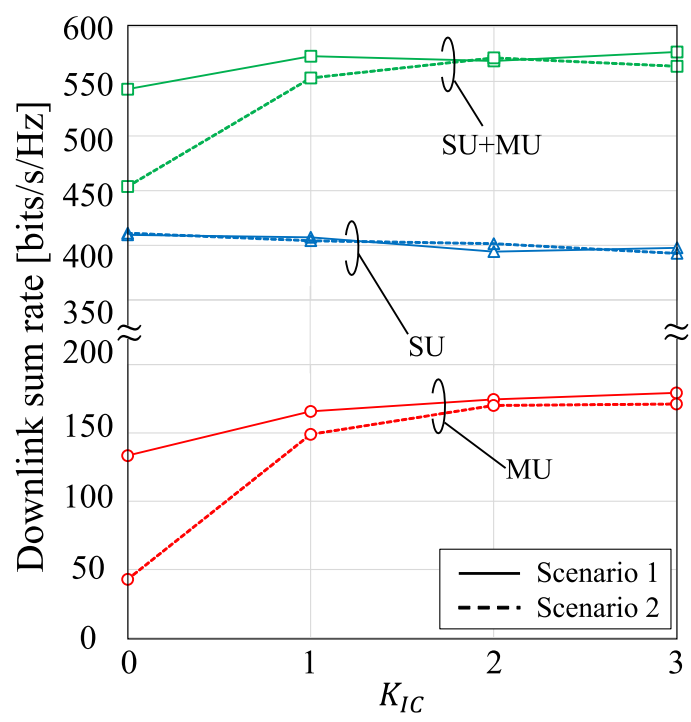

Fig. 3. Downlink sum rate versus $K_{I C}\left(K_{O}=4\right)$.

\section{Conclusion}

In this article, we have proposed a downlink cross-tier interference between MC and SCs. The results show the effectiveness of the proposed approach in terms of total sum-rate in two-tier TDD-HetNets.

\section{Acknowledgments}

This research was supported in part by JSPS KAKENHI (JP17K06427). 